\title{
Intrinsic and Extrinsic Motives Support Adults' Regular Physical Activity Maintenance
}

\section{(ㄷ)(ㄱ) $($ ) $\Theta$}

\author{
Authors \\ Karly Geller, Kate Renneke, Sarah Custer, Grace Tigue
}

\author{
Affiliation \\ Miami University, Kinesiology and Health, Oxford, \\ United States \\ Key words \\ Self-determination theory, Habitual behaviour, Motivation, \\ Physical activity, Maintenance
}

$\begin{array}{lr}\text { received } & 18.12 .2017 \\ \text { revised } & 02.04 .2018 \\ \text { accepted } & 10.04 .2018\end{array}$

Bibliography

DOI https://doi.org/10.1055/a-0620-9137

Sports Medicine International Open 2018; 2: E62-E66

(c) Georg Thieme Verlag KG Stuttgart · New York

ISSN 2367-1890

\author{
Correspondence \\ Dr. Karly Geller \\ Miami University \\ Kinesiology and Health \\ 420 S. Oak Street, Oxford \\ 45056 \\ United States \\ Tel.: + 1/513-529-2702, Fax.: + 1/513-529-5006 \\ gellerks@miamioh.edu
}

\begin{abstract}
Motives for physical activity were compared between adults who either successfully or unsuccessfully maintained regular physical activity over the last 10 years. Adults age 28-45 $(\mathrm{N}=721)$ completed an online survey, reporting their current physical activity levels and self-determination theory (SDT) motives, as well as their physical activity levels at least 10 years prior. With participants' current and retrospective reports of their physical activity, four sample subgroups were created, including maintainers, improvers, decliners, and sedentary. ANOVA analyses were used to examine differences in motives between physical activity maintenance groups. Those who successfully maintained regular physical activity (maintainers) reported higher intrinsic and extrinsic motives compared to those who were not regularly active $(P<0.05)$. Interestingly, maintainers reported similar physical activity motives compared to those who reported increased physical activity over time. Among the current sample and consistent with theory, motives for physical activity significantly influenced participants' long-term maintenance of regular physical activity. Future interventions should consider these constructs to promote sustained physical activity.
\end{abstract}

Regular physical activity is a modifiable behavior that is associated with multiple health benefits [19]. Unfortunately, $47 \%$ of Americans do not meet national recommendations for physical activity and $39 \%$ are completely sedentary $[21,25]$. Moreover, physical activity rates decline as an individual ages $[11,18]$. To date, it remains unclear why some individuals fail to maintain physical activity while others succeed [11]. Specific to physical activity interventions, the majority that targeted long-term maintenance of physical activity have been unsuccessful [20]. Research examining prevalence of successful physical activity maintenance currently remains limited, warranting a better understanding of the associated influences.

One theoretical framework to conceptualize individuals' motivation to sustain regular physical activity is the self-determination theory (SDT). The SDT organizes the internal and external factors that motivate sustainment of health behavior [7]. External or extrinsic factors motivate individuals' physical activity in efforts to achieve external and/or tangible rewards [3]. Internal or intrinsic factors are fueled by internal rewards (e. g., interest) [6], and these internally derived motivations are expected to support long-term habitual behaviors [7]. Deci and Ryan [7] theorized three basic psychological needs required to achieve optimal intrinsic motivation, including competence, relatedness, and autonomy. Competence is an individual's belief they can successfully perform regular physical activity. Relatedness is one's sense of community when physically active and the relationships built from participation. Autonomy is the individual's perceived control over decisions relating to their physical activity. With these needs met through a supportive 
environment, the individual has the potential to develop autonomous and intrinsic motivation [22].

One multidimensional construct of the SDT is physical activity motives, which includes motivations that manifest from both internal and external factors [23]. The first, interest, is an intrinsic motive relative to one's perceptions of interest and fun relative to physical activity. Competence is another intrinsic motive driven by one's motivation to improve and master new physical activity skills and challenges. The first of three extrinsic motives is appearance, which motivates physical activity behaviors via one's desire to develop, improve, and/or maintain their physical physique, muscle definition, and/or body weight. Fitness is the extrinsic motivation to be healthy, strong, and energetic. Finally, social motives are the extrinsic drive to socially interact and meet new people during physical activities. According to the SDT, individuals emphasizing intrinsic motives are expected to maintain healthy behaviors more successfully than those primarily motivated by extrinsic motives [8]. However, evidence regarding how SDT motives strengthen physical activity maintenance remains limited and somewhat ambiguous.

Previous physical activity studies reported that successful physical activity maintenance was supported by both intrinsic and extrinsic motives. Research found the most influential intrinsic motives reported for physical activity maintenance were interest, competence, and fitness [ 1,4$]$. Furthermore, Aaltonen and colleagues [1] reported a decrease in extrinsic motives as participants progressed toward successful physical activity maintenance. Alternatively, similar research found certain extrinsic motives were positively associated with physical activity maintenance [1,4]. Overall, sustained regular physical activity seems to involve multiple motives that are both intrinsic and extrinsic. Research outcomes from this study provide further understanding of adults' motives in relation to their physical activity maintenance. The current study objective was to compare motives between adults who have successfully maintained regular physical activity and those who have not. Based on change from participants' previous and current physical activity, four samples were categorized and compared: maintainers, improvers, decliners, and sedentary. Adults who reported sustained regular physical activity for at least 10 years (maintainers) were expected to have higher psychological needs (autonomy, relatedness, and competence) and intrinsic motives (interest and competence) than participants who remained sedentary and those who reported decreased physical activity (decliners). Similarly, participants who increased their physical activity (improvers) were expected to be similar to maintainers, reporting higher psychological needs and intrinsic motives than decliners and sedentary adults. Differences between sedentary adults and decliners were not expected. Certain extrinsic motives were also expected to be higher among maintainers and improvers, specifically fitness and social motives. All other comparisons were deemed exploratory.

\section{Materials \& Methods}

The current study used a retrospective study design. A one-time assessment was completed by participants. Participants reported their current motives and physical activity levels, and then retrospectively reported their same activity at least 10 years prior. Participants were recruited through a partnership with a university alumni organization in Kansas, USA. Participants were invited to complete the online survey via invitational emails. Approval from the University Institutional Review and participant consent preceded all study procedures. The current study meets the ethical standards of the journal [14].

Data were reported over 4 months in the year 2015. The participant response rate was $20 \%$. Only data collected from adults between the ages of 28 and 45 years were included in the final analysis. Participants were asked to self-report basic demographic information (age, gender, ethnicity, and education), their height, weight, and perceived health status. Researchers used height and weight to calculate each participant's body mass index (BMI) using the following equation: [weight (lb)/height (in) ${ }^{2}$ ] $x 703$. The recommended BMI range for adults is between 19.0 and 24.9 [5]. Current participants with a $\mathrm{BMI} \leq 25.0$ were classified as normal, and participants with a BMI> 25.0 were considered overweight/obese. Perceived health was measured with one question on a 5-point Likert scale, asking participants to report their general health status as poor (1), fair (2), good (3), very good (4), or excellent (5). Physical activity and SDT construct measurements are described in the following sections.

\section{Physical activity measure}

The Godin-Shephard Leisure-Time Physical Activity Questionnaire (GSLTPAQ) was used to assess participants' moderate and strenuous physical activity, which has been previously validated for adults [10-2]. Participants reported the number of previous and current moderate and strenuous physical activity bouts of 15 min they performed in a typical week. Previous physical activity was described with the following statement: "Please recall your usual exercise behavior during college, prior to graduation (when you were approximately 17 to 23 years old)." Similar to their previous physical activity, participants reported their current weekly bouts of moderate and strenuous physical activity.

Self-reported moderate and strenuous physical activity was ultimately calculated into metabolic equivalents (MET). MET represents physical activity in multiples of resting oxygen consumption. Weekly frequency of moderate and strenuous physical activity bouts were weighted using the following calculations: weekly moderate physical activity bouts $x 5$, and weekly strenuous physical activity bouts $x 9$. The MET level required to receive the health benefits of regular physical activity is $\geq 24$ MET per week [10-2]. Based on the change in participants' self-reported previous and current physical activity MET, each participant was categorized into one of the following four sample subgroups: maintainers, or those who met previous and current physical activity recommendations; decliners, or those who met recommendations previously but did not maintain regular activity; sedentary, or participants who never reported meeting physical activity recommendations; and improvers who reported not meeting recommendations previously but currently meeting recommendations.

\section{SDT construct measures}

The Basic Psychological Needs Scale (BPNS) $[8,9]$ was used to assess participants' three basic psychological needs (autonomy, competence, and relatedness). Participants responded to 21 statements using a 7-point Likert scale ranging from 1 ("not at all true for me") to 7 ("very true for me"). This scale has been deemed reliable and 
valid [26]. Participants' current physical activity motives were assessed using the revision of the Motives for Physical Activity Measure (MPAM-R), including competence, interest, appearance, fitness, and social motives [23]. Participants responded to 30 items on a 7-point Likert scale (1, not at all true for me, to 7, very true for me).

\section{Statistical analyses}

Data were analyzed using SPSS Statistics version 23 (IBM, Chicago, IL). Only participants with complete data were included in analyses. Descriptive statistics characterized the sample and described participants' current and previous physical activity MET, BPNS, and physical activity motives. Analysis of variance (ANOVA) F-test was used to test for differences in participants' previous and current physical activity MET, BPNS, and physical activity motives between maintenance subgroup classification (maintainers, improvers, sedentary, and decliners). Any significant difference found between the four subgroups groups $(\mathrm{P}<0.05)$ was then examined using Tukey's HSD post hoc analyses with Bonferroni correction $(0.05 / \mathrm{n}=4$, significance level $=P<0.01)$. Unadjusted and adjusted means were calculated to account for subgroup differences based on the following covariates: gender (male, female) and perceived health (5point Likert scale). Violations of normality and homogeneity of variance were not detected $(P<0.05)$.

\section{Results}

Participants ( $N=721)$ were mostly female (63\%) and Caucasian $(90 \%)$ with a mean age of 33.37 (standard deviation $(S D)=3.87$ ). Participants' average perceived health was $3.83(S D=0.77)$, the mean BMI was $26.00(S D=5.31)$, and $49 \%$ of participants were overweight or obese. Participants were highly educated, including $37 \%$ college graduates and $58 \%$ with a Master's degree or higher. Differences in previous and current physical activity MET between subgroups based on gender, ethnicity, education level, and BMI are shown in $>$ Table 1 . There were no significant differences in previous physical activity MET between subgroups. Males reported significantly higher current physical activity MET than females (mean difference $(M D)=10.84, P<0.001)$. Overweight/obese participants reported significantly lower physical activity MET than those with a normal BMI (MD = 5.06, $\mathrm{P}<0.05)$.

Comparison of physical activity and self-determination constructs between maintenance subgroups are provided in $>$ Table 2 . Significant covariates were gender $(P<0.001)$ and perceived health $(\mathrm{P}<0.001)$. As seen, previous and current physical activity MET were different between maintenance groups $(P<0.05)$. Pairwise comparisons were used to reveal specific subgroup differences $(\mathrm{P}<0.01)$. Maintainers reported higher previous weekly strenuous and moderate physical activity MET compared to improvers $(\mathrm{MD}=64.46, \mathrm{P}<0.001)$ and sedentary participants $(\mathrm{MD}=66.65$, $\mathrm{P}<0.001)$. Participants who improved their physical activity reported lower previous MET than those who declined (MD $=53.81$, $P<0.001)$. Finally, decliners reported more previous physical activity MET than sedentary participants (MD =56.01, $\mathrm{P}<0.001)$. Specific to participants' current weekly strenuous and moderate physical activity, maintainers reported higher MET than improvers ( $M D=11.96, P<0.001)$, decliners $(M D=49.13, P<0.001)$, and the sedentary $(M D=50.49, P<0.001)$. And, improvers reported higher current physical activity MET than decliners $(\mathrm{MD}=37.17, \mathrm{P}<0.001)$ and sedentary participants (MD $=39.53, \mathrm{P}<0.001)$.

Also shown in $>$ Table 2 are differences in physical activity motives between maintenance subgroups. Except for the appearance motive, subgroup differences were detected for all other motives $(P<0.05)$. Significant covariates were gender $(P<0.05)$ and perceived health $(P<0.001)$. Pairwise comparisons revealed maintainers reported higher physical activity interest than decliners $(\mathrm{MD}=1.00, \mathrm{P}<0.001)$ and sedentary participants $(\mathrm{MD}=1.33$, $P<0.001)$. Improvers were higher in interest compared to sedentary participants $(\mathrm{MD}=0.84, \mathrm{P}<0.001)$. Maintainers were higher in competence compared to improvers ( $\mathrm{MD}=0.57, \mathrm{P}<0.001)$, decliners $(M D=1.28, P<0.001)$, and the sedentary $(M D=1.58$, $\mathrm{P}<0.001)$, and improvers were higher in competence compared to decliners $(\mathrm{MD}=0.72, \mathrm{P}<0.001)$ and sedentary participants $(M D=1.01, P<0.001)$. Similar differences were seen in extrinsic motives between maintenance subgroups. Maintainers reported higher fitness motive compared to decliners $(\mathrm{MD}=0.67, \mathrm{P}<0.001)$ and sedentary participants $(M D=0.55, P<0.001)$, improvers were higher in fitness than decliners ( $M D=0.51, P<0.001)$. Last, social motive was higher among maintainers compared to decliners $(M D=0.45$, $\mathrm{P}<0.001)$ and sedentary participants $(\mathrm{MD}=0.69, \mathrm{P}<0.001)$.

\section{Discussion}

The current study examined the relationship between certain SDT constructs and physical activity maintenance. This study supports

- Table 1 Adjusted mean differences in metabolic equivalent of physical activity between demographic subgroups.

\begin{tabular}{|c|c|c|c|}
\hline \multirow{2}{*}{$\begin{array}{l}\text { Sample } \\
\text { Characteristics }\end{array}$} & \multirow[t]{2}{*}{ n (\%) } & $\begin{array}{c}\text { Previous PA } \\
\text { MET }\end{array}$ & $\begin{array}{c}\text { Current PA } \\
\text { MET }\end{array}$ \\
\hline & & Mean (SEM) & Mean (SEM) \\
\hline Total Sample & $721(100 \%)$ & $61.81(1.72)$ & $46.48(1.26)$ \\
\hline \multicolumn{4}{|l|}{ Gender } \\
\hline Male & $268(37 \%)$ & $65.54(2.85)$ & $53.30(2.12)$ \\
\hline Female & $453(63 \%)$ & $59.61(2.15)$ & $42.46(1.53)$ \\
\hline \multicolumn{4}{|l|}{ Ethnicity } \\
\hline White & $650(90 \%)$ & $60.71(1.82)$ & $46.37(1.34)$ \\
\hline Mixed or Other & $71(10 \%)$ & $71.93(5.03)$ & $47.54(3.53)$ \\
\hline \multicolumn{4}{|l|}{ Education } \\
\hline HS Diploma or AS & $27(4 \%)$ & $57.67(10.05)$ & $38.78(6.78)$ \\
\hline College Graduate & $276(38 \%)$ & $63.06(2.91)$ & $45.51(2.04)$ \\
\hline Advanced Degree & $418(58 \%)$ & $61.26(2.17)$ & $46.49(1.26)$ \\
\hline \multicolumn{4}{|l|}{$\mathrm{BMI}$} \\
\hline †Normal & 365 (51\%) & $62.90(2.44)$ & $48.99(1.90)$ \\
\hline $\begin{array}{l}\text { §Overweight/ } \\
\text { Obese }\end{array}$ & $356(49 \%)$ & $60.70(2.42)$ & $43.93(1.64)$ \\
\hline \multicolumn{4}{|c|}{ 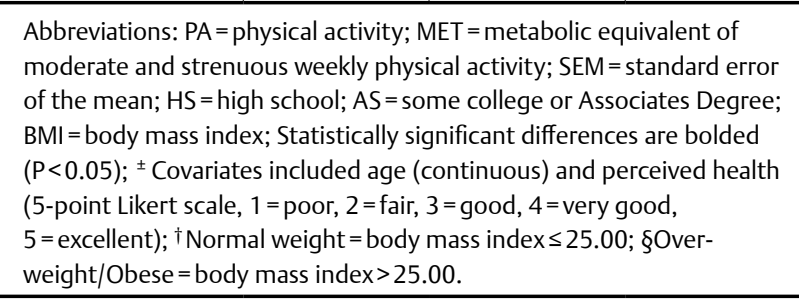 } \\
\hline
\end{tabular}




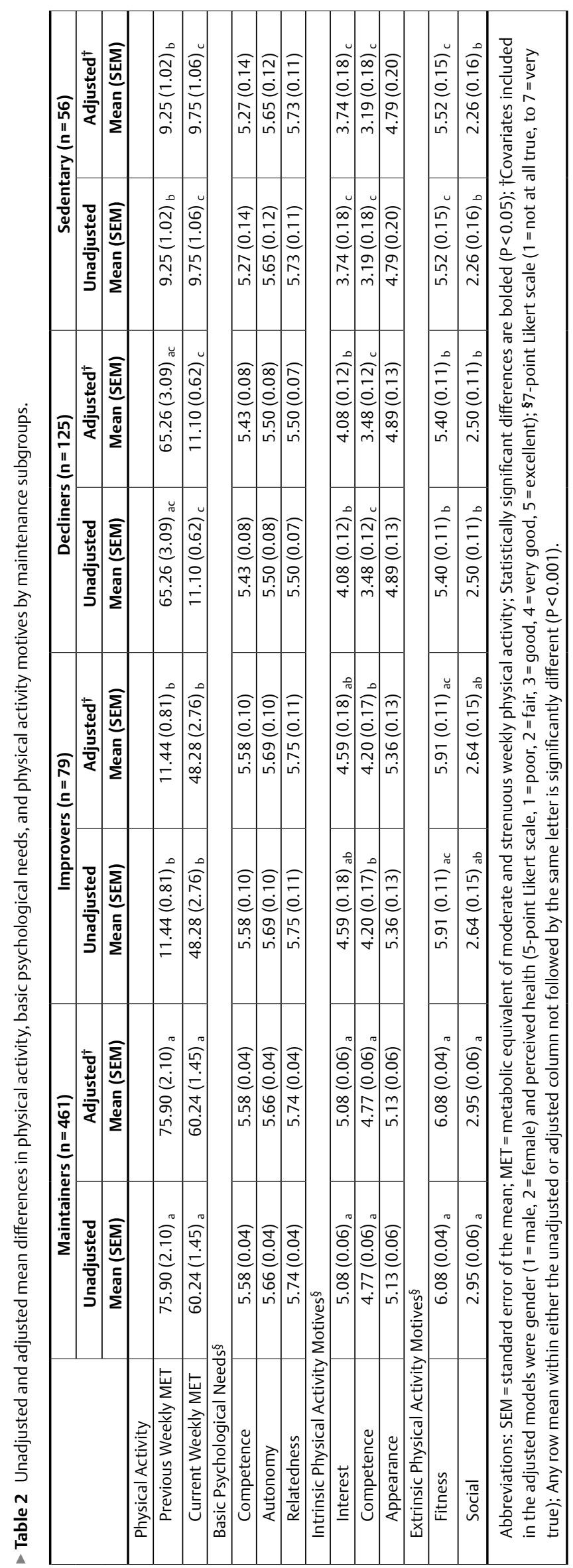

previous research and theory, and also adds to the current literature. To begin, males in the current study reported more current physical activity than their female counterparts, which mimics outcomes of previous research [12]. Also as expected, participants with a $\mathrm{BMI} \leq 25.00$ reported more current physical activity than those who were overweight or obese [13]. Secondly, differences in previous and current physical activity followed expected patterns and confirmed the categorization of maintenance groups.

Relative to SDT constructs, no differences emerged in basic psychological needs between the maintenance groups. According to theory, this indicates all current participants had the psychological framework to facilitate successful maintenance of regular physical activity [8]. Maintainers in the current study reported higher intrinsic motives (competence and interest) and extrinsic motives (fitness and social) than decliners and sedentary participants. This is comparable to evidence indicating that both aid in successful physical activity maintenance $[1,4,5]$. Interestingly, no differences were seen between maintainers and improvers, which may indicate similar motives when both adopting and maintaining physical activity.

Specific differences in physical activity motives between maintenance groups are comparable to previous research. Physical activity maintainers were higher in competence than all other maintenance subgroups, confirming similar research [1,4]. Moreover, competence was the only motive maintainers reported higher than improvers; hence, competence-related motives for physical activity may bridge a gap between adoption and successful maintenance. Compared to decliners and sedentary participants, maintainers reported higher interest for physical activity; and improvers reported more interest than the sedentary. These results are comparable to previous studies, reporting highest interest among adults who had successfully maintained physical activity $[4,5]$.

Extrinsic motives were also highest among maintainers and improvers. Both maintainers and improvers reported a higher fitness motive than decliners, which supports evidence that health and fitness motives predict intrinsic motivation [15]. Maintainers also reported the highest social motive, confirming evidence that social motivations can facilitate intrinsic regulation of physical activity [16]. Interestingly, no differences in appearance motive were seen between maintenance groups. Buckworth and others [4] found appearance motives were highest among college students maintaining physical activity. Conversely, appearance was not a salient motivation for physical activity maintenance among a sample of young to middle-aged adults [17]. Also, appearance motives were inversely related to female adults' physical activity [24]. Further research is needed to clarify certain developmental shifts that impact one's motives to sustain regular physical activity.

\section{Conclusions}

Given the numerous health benefits of regular physical activity, a better understanding of the mechanisms driving long-term, habitual physical activity behavior is required. Based on current outcomes, both intrinsic and extrinsic motivations impact habitual physical activity. Additional research is needed to clarify possible age/developmental differences to determine the motives most conducive to physical activity maintenance across the lifespan. One 
study limitation was the homogeneous study sample. Most participants were Caucasian and highly educated, which may not generalize to the greater population. Also, the current study used a retrospective study design that is open to memory bias. Prospective, longitudinal examinations are warranted.

\section{Conflicts of Interest}

The authors declare that they have no conflict of interest

\section{References}

[1] Aaltonen S, Leskinen T, Morris T, Alen M, Kaprio J, Liukkonen J, Kujala UM. Motives for and barriers to physical activity in twin pairs discordant for leisure time physical activity for 30 years. Int J Sports Med 2012; 3: 157-163

[2] Amireault S, Godin G. The Godin-Shephard leisure-time physical activity questionnaire: Validity evidence supporting its use for classifying healthy adults into active and insufficiently active categories. Percept Mot Skills 2015; 120: 604-622

[3] Brown HD. Principles of Language Learning and Teaching. 5th ed. White Plains, NY: Pearson Education; 2006

[4] Buckworth J, Lee RE, Regan G, Schneider LK, DiClemente CC. Decomposing intrinsic and extrinsic motivation for exercise: Application to stages of motivational readiness. Psychol Sport Exerc 2007; 8: 441-461

[5] Center for Disease Control and Prevention, 2012. Defining overweight and obesity. Retrieved from http://www.cdc.gov/obesity/adult/ defining.html

[6] Coon D, Mitterer JO. Introduction to Psychology: Gateways to Mind and Behavior with Concept Maps. Belmont, CA: Wadsworth; 2010

[7] Deci E, Ryan R. Intrinsic Motivation and Self-Determination in Human Behavior. New York: Plenum; 1985

[8] Deci EL, Ryan RM. The "what" and "why" of goal pursuits: Human needs and the self determination of behavior. Psychological Inquiry 2000; 11: 227-268

[9] Deci EL, Ryan RM, Gagné M, Leone DR, Usunov J, Kornazheva BP. Need satisfaction, motivation, and well-being in the work organizations of a former Eastern Bloc country. Pers Soc Psychol Bull 2001; 27: 930-942

[10] Godin G, Shephard R. Godin leisure-time exercise questionnaire. Med Sci Sports Exerc 1997; 29: S36

[11] Gordon-Larsen Nelson MC, Popkin BM. Longitudinal physical activity and sedentary behavior trends. Am J Prev Med 2004; 27: 277-283
[12] Hagströmer M, Troiano RP, Sjöström M, Berrigan D. Levels and patterns of objectively assessed physical activity: A comparison between Sweden and the United States. Am J Epidemiol 2010; 10: 1055-1064

[13] Hansen BH, Holme I, Anderssen SA, Kolle E. Patterns of objectively measured physical activity in normal weight, overweight, and obese individuals (20-85 years): A cross-sectional study. PLoS One 2013; 8 : e53044

[14] Harriss D], Macsween A, Atkinson G. Standards for ethics in sport and exercise science research: 2018 update. Int J Sports Med 2017; 38: $1126-1131$

[15] Ingledew DK, Markland D, Ferguson E. Three levels of exercise motivation. Applied Psychol Health Well Being 2009; 1: 336-355

[16] Ingledew DK, Markland D. The role of motives in exercise participation. Psychol Health 2008; 23: 807-828

[17] Ingledew DK, Markland D, Medley AR. Exercise motives and stages of change. J Health Psychol 1998; 3: 477-489

[18] Larouche R, Laurencelle L, Shephard RJ, Trudeau F. Life transitions in the waning of physical activity from childhood to adult life in the Trois-Rivières Study. J Phys Act Health 2012; 9: 516-524

[19] Lee IM, Shiroma E], Lobelo F, Puska P, Blair SN, Katzmarzyk PT. Lancet Physical Activity Series Working Group. Effect of physical inactivity on major non-communicable diseases worldwide: An analysis of burden of disease and life expectancy. Lancet 2012; 380: 219-229

[20] Muller-Riemenschneider F, Reinhold T, Nocon M, Willich SN. Long-term effectiveness of interventions promoting physical activity: A systematic review. J Prev Med 2008; 47: 354-368

[21] Pleis JR, Lucas JW. Summary health statistics for U.S. adults: National Health Interview Survey, 2007. National Center for Health Statistics. Vital Health Stat 102009

[22] Ryan RM, Deci EL. Self-determination theory and the facilitation of intrinsic motivation, social development, and wellbeing. Am Psychol 2000; 55: 68-78

[23] Ryan RM, Frederick CM, Lepes D, Rubio N, Sheldon KM. Intrinsic motivation and exercise adherence. Int J Sport Psychol 1997; 28 : 335-354

[24] Segar ML, Spruijt-Metz D, Nolen-Hoeksema S. Go figure? Body-shape motives are associated with decreased physical activity participation among midlife women. Sex Roles 2006; 54: 175-187

[25] Tucker JM, Welk G], Beyler NK. Physical activity in US adults: Compliance with the physical activity guidelines for Americans. Am J Prev Med 2011; 40: 454-461

[26] Vlachopoulos SP, Ntoumanis N, Smith AL. The basic psychological needs in exercise scale: Translation and evidence for cross-cultural validity. Int J Sport Exerc Psychol 2010; 8: 394-412 